\title{
Anaplastic large cell lymphoma of the vertebra masquerading as tuberculous spondylitis: potential pitfalls of conventional imaging
}

\author{
Lih Kin Khor $\cdot$ Shi Wang $\cdot$ Suat-Jin Lu
}

Received: 17 August 2012/Accepted: 22 September 2012/Published online: 5 October 2012

(C) SIMI 2012

\section{Case report}

A 26-year-old woman presented with a 6-month history of progressive neck pain associated with a 1-month history of fever, cough, and weight loss. Physical examination revealed a febrile patient with a temperature of $39.1{ }^{\circ} \mathrm{C}$. There were localized tenderness and reduced range of motion at the cervicothoracic region of the spine. There was no neurological deficit. Laboratory findings revealed a raised white blood cell count of $21.6 \times 10^{9} / \mathrm{L}$. There was elevation of inflammatory markers such as erythrocyte sedimentation rate $(79 \mathrm{~mm} / \mathrm{hr})$ and C-reactive protein $(137 \mathrm{mg} / \mathrm{L})$. Serum lactate dehydrogenase and tumor markers such as alpha-fetoprotein, beta-human chorionic gonadotropin, carcinoembryonic antigen, and CA-125 levels were normal.

Radiographs of the cervical spine and chest revealed bony erosions in the $\mathrm{C} 7$ and $\mathrm{T} 1$ vertebrae (Fig. 1, arrowhead), and right upper lobe consolidation with air bronchograms (Fig. 1, arrow). Contrast-enhanced computed tomography (CT) scan showed bony erosions with pathological fractures involving the $\mathrm{C} 7$ to $\mathrm{T} 3$ vertebrae (Fig. 2a, $\mathrm{b}$, arrows). An adjacent large paravertebral thick-walled rim-enhancing lesion was seen (Fig. 2c, arrow). There was

L. K. Khor $(\bowtie) \cdot$ S.-J. Lu

Department of Diagnostic Imaging, National University

Hospital, 5 Lower Kent Ridge Road,

Singapore 119074, Singapore

e-mail: superkhor@hotmail.com

S. Wang

Department of Pathology, National University Hospital,

Singapore, Singapore

e-mail: shi_wang@nuhs.edu.sg lung consolidation in the posterior segment of the right upper lobe (Fig. 2d, arrow) as well as prominent mediastinal and retroperitoneal (Fig. 2e, arrow) lymph nodes.

Gadolinium-enhanced magnetic resonance imaging (MRI) of the spine was performed to evaluate the soft tissues and spinal cord. MRI revealed altered marrow signal and abnormal enhancement in the $\mathrm{C} 7$ to $\mathrm{T} 4$ vertebrae with adjacent enhancing subligamentous and epidural masses as well as mild kyphosis (Fig. 3, arrows). There was relative sparing of most of the intervertebral disks. The radiographic, $\mathrm{CT}$, and MRI findings were typical of pulmonary tuberculosis (TB) with tuberculosis spondylitis. The clinical findings further corroborated this presumptive diagnosis. However, multiple induced sputum specimens were negative for acid-fast bacilli smears and molecular TB tests. Moreover, the vertebral destruction seemed more extensive than expected for TB that had been symptomatic for only 6 months. Hence the possibility of an alternative diagnosis was entertained.

$\left[{ }^{18} \mathrm{~F}\right]$ fluorodeoxyglucose positron emission tomography/ computed tomography (FDG-PET/CT) scan (Biograph mCT 64-slice, Siemens AG, Erlangen, Germany) was subsequently performed to assess the extent and pattern of disease, and to determine the most suitable site for biopsy. The PET/CT scan showed FDG-avid vertebral and paravertebral masses (standardized uptake value, SUVmax 28.3) extending from the $\mathrm{C} 7$ to $\mathrm{T} 4$ vertebrae (Fig. 4a, arrow), FDG-avid lung consolidation with air bronchograms (SUVmax 7.9) in the right upper lobe (Fig. 4b, arrows), FDG-avid lesions in both kidneys (SUVmax 11.1) (Fig. 4c, arrows), and FDG-avid retroperitoneal (SUVmax 7.6) lymphadenopathy (Fig. 4d, arrows). In addition, the spleen (SUVmax 8.3) and bone marrow (SUVmax 5) showed diffusely increased FDG uptake (Fig. 4a, e, arrowheads). The pattern of disease demonstrated on 
Fig. 1 Radiographs of the cervical spine and chest revealed bony erosions in the $\mathrm{C} 7$ and $\mathrm{T} 1$ vertebrae accompanied by right upper lobe consolidation arrows
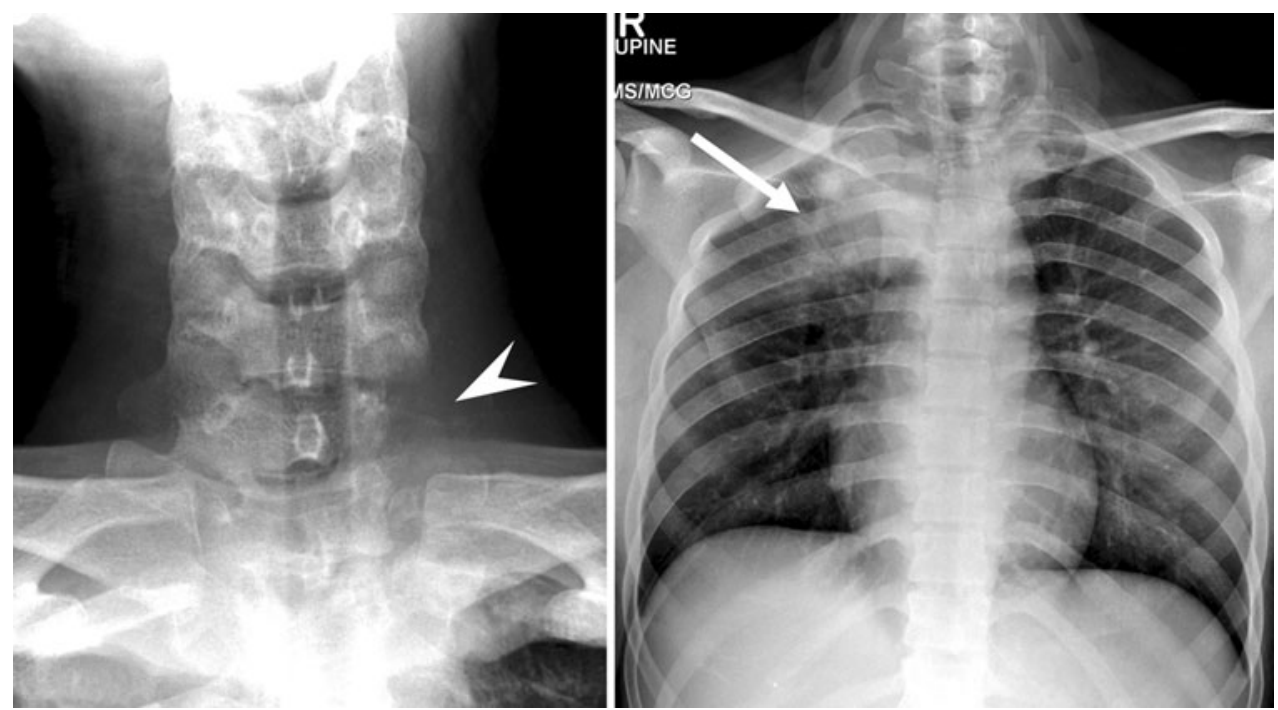
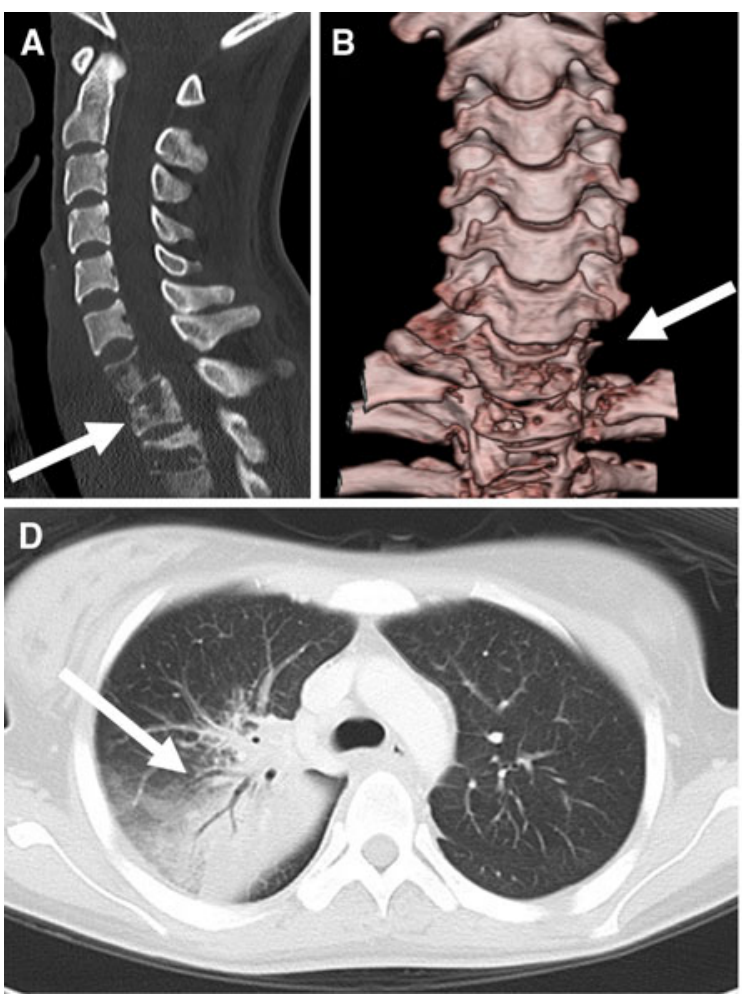

Fig. 2 Pathological fractures involving the C7 to T3 vertebrae (a and b). An adjacent large paravertebral thick-walled rim-enhancing lesion was seen (c). Lung consolidation in the posterior segment of the right

FDG-PET/CT supported a lymphoproliferative disorder rather than $\mathrm{TB}$.

A core biopsy of the $\mathrm{C} 7$ vertebra was performed. The biopsy specimens show diffuse sheet-like infiltrates of large neoplastic lymphoid cells with large vesicular nuclei, single to multiple conspicuous nucleoli, and a moderate amount of pale cytoplasm (Fig. 5a). Scattered neoplastic cells with kidney-shaped nuclei resembling hallmark cells are also
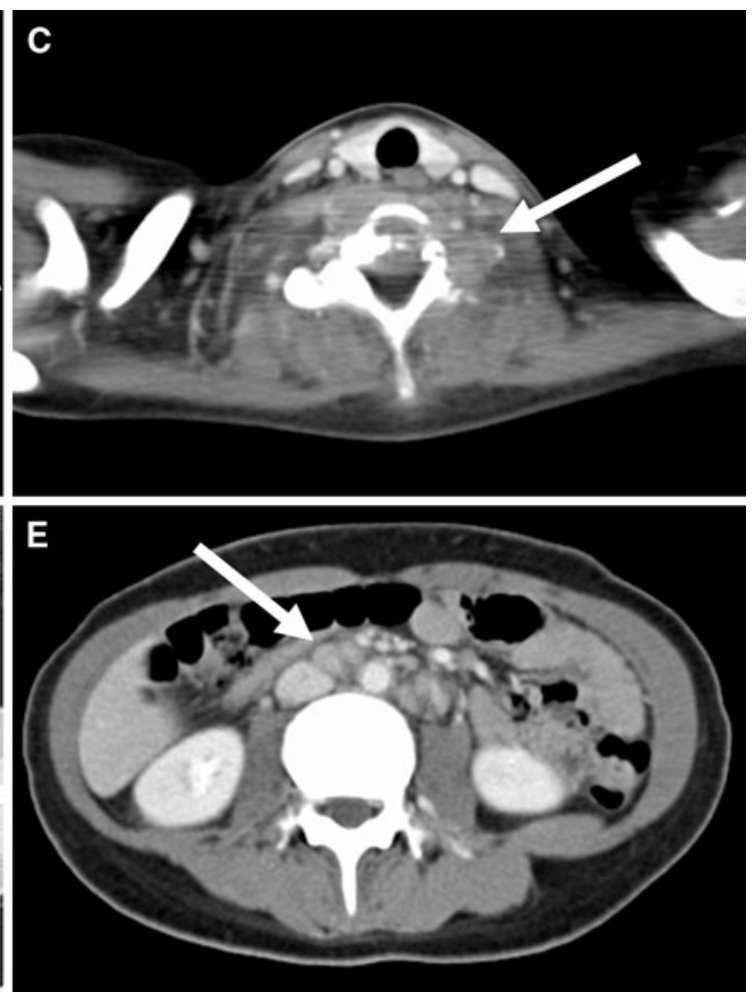

upper lobe (d) and prominent mediastinal and retroperitoneal (e) lymph nodes were also noted

identified. Immunohistochemical stains were performed. The neoplastic lymphoid cells show positive staining for CD4, CD43, CD30, and ALK1 (Fig. 5b). Other immunohistochemical stains CD20, CD79A, CD15, CD2, CD3, $\mathrm{CD} 5, \mathrm{CD} 7$, and CD8 were all negative. The diagnosis on the biopsy specimens is that of an anaplastic large cell lymphoma (ALCL), ALK positive. The majority of ALCL, ALK positive cases have the $\mathrm{t}(2: 5) / \mathrm{NPM}-\mathrm{ALK}$ translocation 
Fig. 3 MRI reveals altered marrow signal and abnormal enhancement in the $\mathrm{C} 7$ to $\mathrm{T} 4$ vertebrae with adjacent enhancing subligamentous and epidural masses as well as mild kyphosis
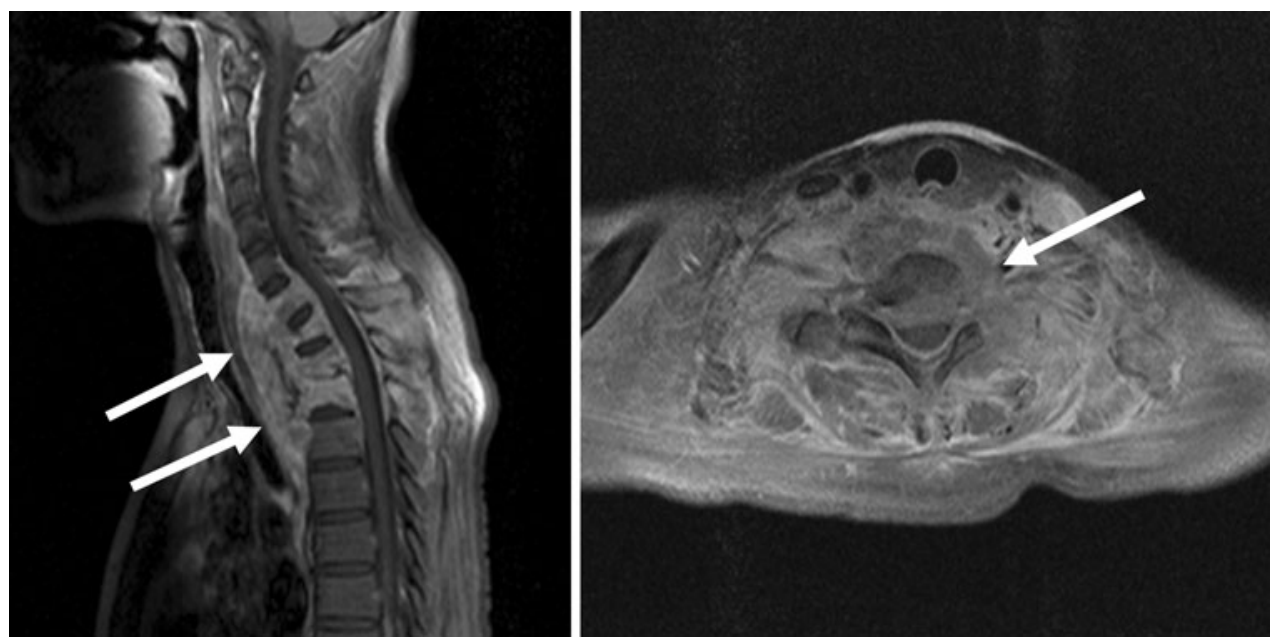
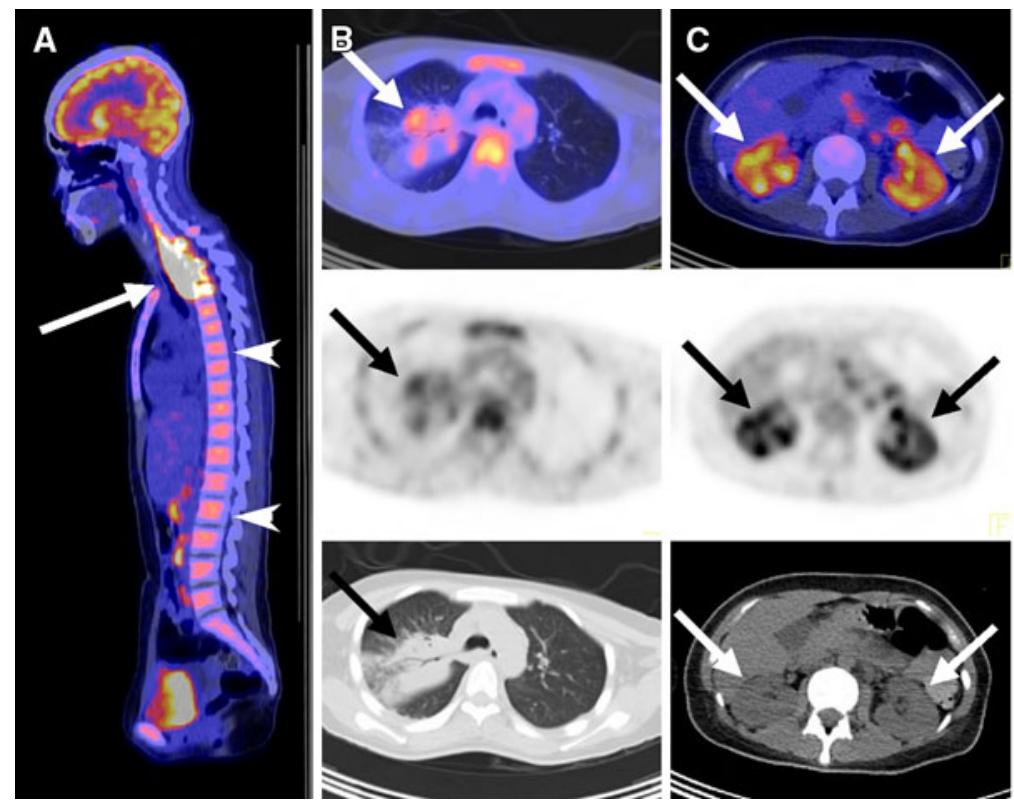
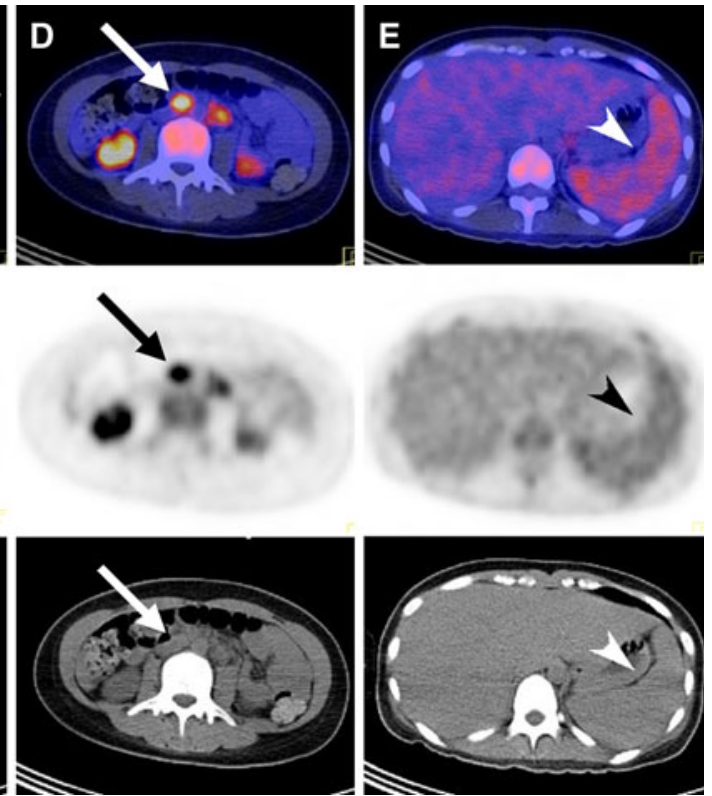

Fig. 4 PET/CT scan shows FDG-avid vertebral and paravertebral masses (standardized uptake value, SUVmax 28.3) extending from the C7 to T4 vertebrae (a). FDG-avid lung consolidation (SUVmax 7.9) in the right upper lobe (b), FDG-avid lesions in both kidneys

that demonstrates both positive cytoplasmic and nuclear staining with ALK1 immunostain. In cases with variant translocations, as in this case, the ALK1 staining is membranous and cytoplasmic. Further fluorescence in situ hybridization (FISH) molecular analysis performed confirms the presence of rearrangements involving the ALK gene. Thus there was a resultant change of diagnosis from TB spondylitis with pulmonary TB to ALCL with nodal (above and below diaphragm) and extranodal (lungs, bones, kidneys, and spleen) involvement. The patient underwent a C4 to T6 spinal decompression and stabilization surgery followed by chemotherapy. Interim and end of treatment
(SUVmax 11.1) (c), and FDG-avid retroperitoneal (SUVmax 7.6) lymphadenopathy (d). In addition, the spleen (SUVmax 8.3) and bone marrow (SUVmax 5) show diffusely increased FDG uptake (a and e)

PET/CT show a complete metabolic resolution of all nodal and extranodal lesions (Fig. 6).

\section{Discussion}

ALCL is a neoplasia of T-cell or null-cell origin. Bony involvement by ALCL, either as a primary or secondary site of spread, is rare [1]. TB is a prevalent granulomatous infection. Bony involvement accounts for approximately $5 \%$ of extrapulmonary TB, of which vertebral involvement accounts for approximately $50 \%$ of them $[2,3]$. Both 


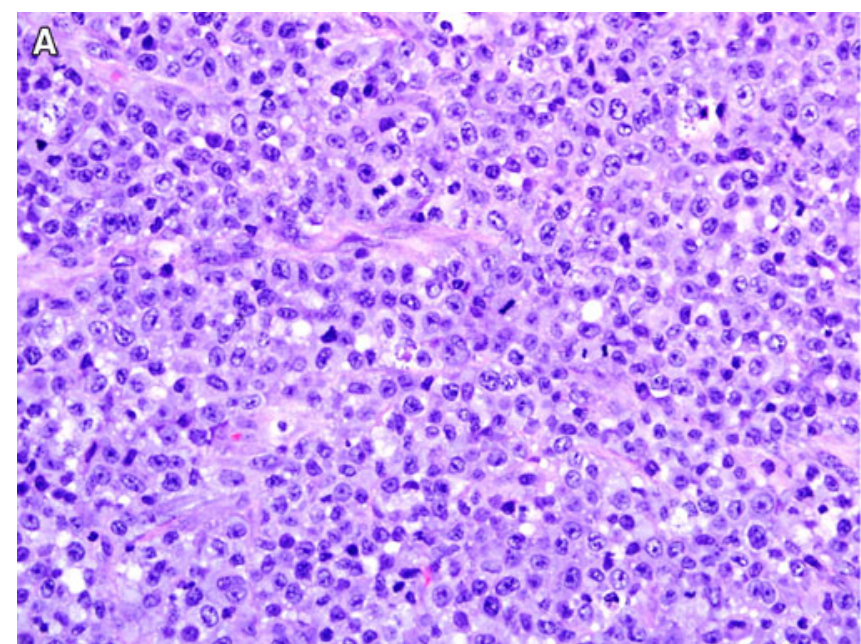

Fig. 5 The biopsy specimens show diffuse sheet-like infiltrates of large neoplastic lymphoid cells with large vesicular nuclei, single to multiple conspicuous nucleoli, and moderate amount of pale

Fig. 6 Interim and end of treatment $\mathrm{PET} / \mathrm{CT}$ show a complete metabolic resolution of all nodal and extranodal lesions

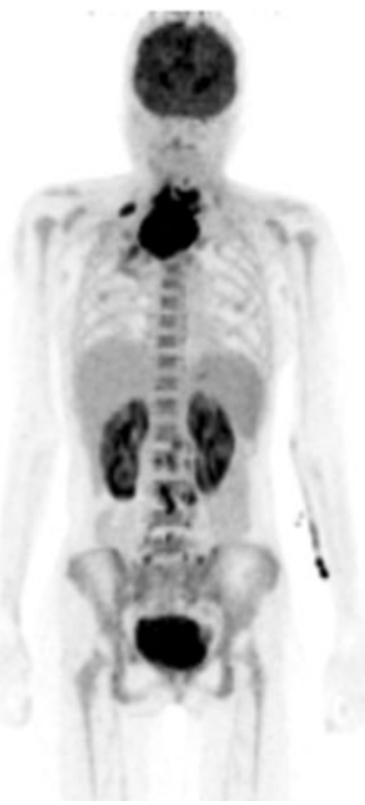

TB and ALCL can involve different organs and are known to be mimickers of disease with a few reports of the two entities masquerading as the other, particularly in the form of vertebral involvement $[4,5]$.

Patients presenting with neck pain are often evaluated with conventional imaging such as radiography, CT, and MRI. The classic appearance of TB spondylitis on conventional imaging is involvement of two or more contiguous vertebrae with associated paravertebral mass or collection. There is usually a relative sparing of the intervertebral disks until the disease is advanced [2, 3]. This classic imaging appearance is due to hematogenous spread of Mycobacterium tuberculosis to the vertebral column with formation of TB abscesses. Extension of infection to

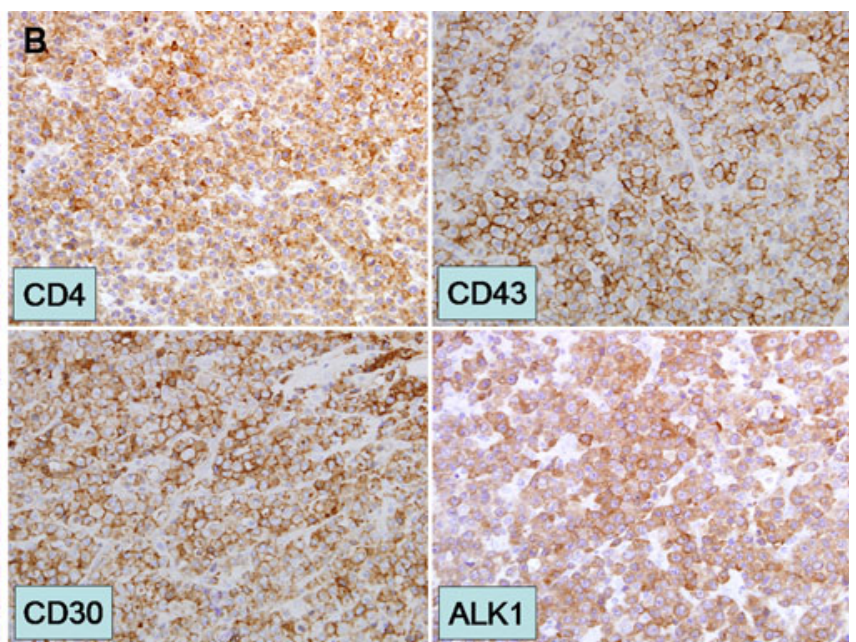

cytoplasm (a). Scattered neoplastic cells with kidney-shaped nuclei resembling hallmark cells are also identified. The neoplastic lymphoid cells show positive staining for CD4, CD43, CD30, and ALK1 (b)
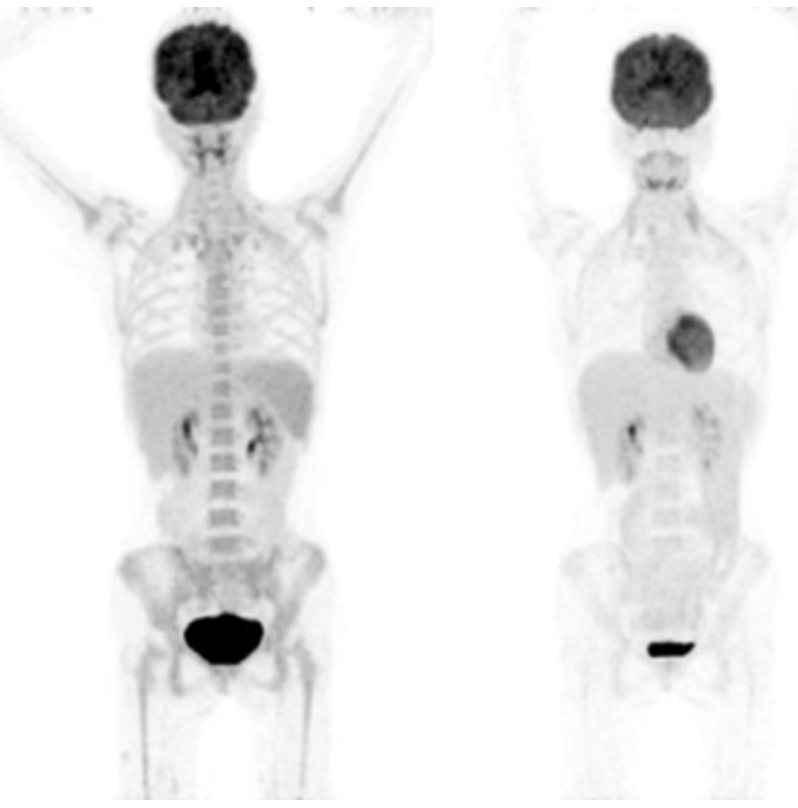

the paravertebral soft tissues leads to formation of paravertebral abscesses. From there, the infection tracks in a typical subligamentous fashion beneath the anterior and posterior longitudinal ligaments to the neighboring vertebrae, resulting in contiguous vertebral involvement. Progressive vertebral destruction leads to multiple consecutive vertebral collapses, eventually resulting in kyphosis and gibbus formation $[2,3]$. This typical imaging appearance of TB spondylitis was present in this patient. The concurrent right upper lobe lung consolidation, suggestive of pulmonary TB, further strengthened the overall diagnosis of TB. However, despite the typical imaging appearance of TB spondylitis, definitive histopathological or microbiological diagnosis should be actively pursued whenever 
possible, as in this case. Repeated negative microbiological studies of induced sputum or bronchoscopic specimens especially from a dense consolidation should prompt the possibility of an alternative diagnosis to TB. This is especially so when the vertebral destruction seemed more extensive than expected for TB. In such a situation, addition of FDG-PET/CT to conventional imaging is valuable as FDG-PET/CT is able to demonstrate the extent and pattern of disease, and determine the most suitable site for biopsy. In this patient, the pattern of disease on FDG-PET/ CT with spleen and marrow abnormalities, along with lymphadenopathy above and below the diaphragm, favored a lymphoproliferative disorder ahead of TB. Furthermore, FDG-PET/CT is able to detect lesions that are occult on conventional imaging as metabolic changes often precede morphological changes. The kidney, spleen, and marrow abnormalities demonstrated on FDG-PET/CT were occult on contrast-enhanced $\mathrm{CT}$ in this patient. Overall, FDG$\mathrm{PET} / \mathrm{CT}$ is able to provide incremental value to conventional imaging in the diagnostic work-up, which can potentially expedite diagnosis and treatment. However, if there is a significant delay in obtaining the definitive diagnosis, starting TB treatment empirically should be considered.

In conclusion, this case highlights a potential pitfall of conventional imaging in differentiating TB spondylitis from vertebral lymphoma and the incremental value that FDG-PET/CT provides. This case also highlights a useful learning point: while it is tempting to base the final diagnosis on typical imaging appearance and start treatment early with the aim of expediting care, definitive histopathological or microbiological diagnosis should be actively pursued whenever possible.

Conflict of interest None

\section{References}

1. Pant V, Jambhekar NA, Madur B, Shet TM, Agarwal M, Puri A et al (2007) Anaplastic large cell lymphoma (ALCL) presenting as primary bone and soft tissue sarcoma-a study of 12 cases. Indian J Pathol Microbiol 50:303-307

2. Shikhare SN, Singh DR, Shimpi TR, Peh WC (2011) Tuberculous osteomyelitis and spondylodiscitis. Semin Musculoskelet Radiol $15: 446-458$

3. Garg RK, Somvanshi DS (2011) Spinal tuberculosis: a review. J Spinal Cord Med 34:440-454

4. Stockdale AD, Jones C, Watkins RM (1988) Pott's disease masquerading as vertebral lymphoma. Br J Clin Pract 42:389-391

5. Huang B, Li CQ, Liu T, Zhou Y (2009) Primary non-Hodgkin's lymphoma of the lumbar vertebrae mimicking tuberculous spondylitis: a case report. Arch Orthop Trauma Surg 129:1621-1625 